\title{
Lansoprazole Treatment of Patients With Chronic Idiopathic Laryngitis: A Placebo-Controlled Trial
}

\author{
Hashem B. El-Serag, M.D., M.P.H., Patty Lee, M.D., Anna Buchner, M.D., John M. Inadomi, M.D., \\ Michael Gavin, M.D., and Denis M. McCarthy, M.D., M.Sc. \\ Divisions of Gastroenterology and Health Services Research, Houston VA Medical Center and Baylor \\ College of Medicine, Houston, Texas; Otolaryngology Department and Division of Gastroenterology, \\ Albuquerque VA Medical Center and University of New Mexico, Albuquerque, New Mexico; and Divisions of \\ Gastroenterology and Health Services Research, Ann Arbor VA Medical Center and University of Michigan, \\ Ann Arbor, Michigan
}

OBJECTIVE: Previous uncontrolled studies suggested a therapeutic benefit for treating gastroesophageal reflux disease (GERD) among patients with laryngitis. The present study is the first randomized, placebo-controlled, double-blind study of gastric acid suppression among patients with laryngitis in the United States.

METHODS: Patients diagnosed with idiopathic chronic laryngitis were randomized to receive either lansoprazole 30 $\mathrm{mg}$ p.o. b.i.d. or a matching placebo for 3 months. Before randomization, all patients underwent upper endoscopy, dual probe ambulatory 24-h esophageal $\mathrm{pH}$-metry, and laryngoscopy, as well as completing a symptom questionnaire for GERD and laryngitis. The primary outcome of treatment was the complete resolution of laryngeal symptoms.

RESULTS: A total of 22 patients with symptoms and signs of chronic laryngitis were enrolled, 20 of whom completed the study. At baseline, there were no significant differences between the two groups with regards to GERD symptoms, erosive esophagitis, proximal and distal esophageal $\mathrm{pH}$ metry, or laryngeal signs and symptoms. In an intention-totreat analysis, six patients in the lansoprazole group (50\%) and only one patient $(10 \%)$ in the placebo group achieved a complete symptomatic response, $p=0.04$. Apart from receiving lansoprazole, there were no significant differences between responders and nonresponders in any of baseline esophageal or laryngeal signs and symptoms.

CONCLUSIONS: Empirical treatment with lansoprazole is efficacious in relieving symptoms of laryngitis compared to placebo. Such treatment can be considered as a first-line option in managing patients with idiopathic chronic laryngitis. (Am J Gastroenterol 2001;96:979-983. (c) 2001 by Am. Coll. of Gastroenterology)

\section{INTRODUCTION}

Several lines of evidence indicate that patients with gastroesophageal reflux disease (GERD) have an increased risk of developing concomitant laryngeal disorders (1-3). GERDrelated laryngitis is thought to affect preferentially the posterior larynx and to manifest as lesions ranging in severity from swelling of the vocal cords to severe ulceration, granuloma formation, or laryngeal stenosis (4).

In a case-control study of more than 200,000 hospitalized veterans, there was an $80-100 \%$ increase in the risk of aphonia, laryngitis, and laryngeal stenosis among patients with erosive esophagitis as compared with controls without esophagitis (1). In a clinical series, a large proportion (up to $78 \%$ ) of patients with persistent symptoms of chronic laryngitis for more than $3 \mathrm{wk}$ were found to have increased esophageal acid exposure on ambulatory 24-h esophageal pH-metry $(3,5)$. However, classical symptoms of GERD are absent in more than half of the patients with suspected GERD-related laryngitis (3-6). In addition, endoscopic evidence of erosive esophagitis is absent in most patients suspected of having GERD-induced laryngitis (7).

Uncontrolled studies suggested a beneficial role for antireflux therapy in the management of patients with chronic laryngitis (8-11). For example, Kamel et al. (8) reported on 182 patients with chronic laryngitis and concomitant GERD symptoms in whom GERD was treated in a stepwise fashion. Almost half of the patients responded to lifestyle modification, whereas the rest received a 6-wk course of famotidine $20 \mathrm{mg}$ b.i.d. followed sequentially by omeprazole 20 $\mathrm{mg}, 40 \mathrm{mg}$, and $80 \mathrm{mg}$ daily for nonresponders. Eventually, in the majority, laryngitis healed or improved (8). The type of therapy used as well as the dose and duration of treatment were variable among different studies $(8-11)$. However, laryngitis may resolve spontaneously, so the benefit from antireflux treatment is hard to assess in many of these studies because of the lack of appropriate controls. Thus, more controlled trials are needed. We carried out a small, randomized, double-blind, placebo-controlled study to assess the potential benefit of gastric acid suppression in patients with idiopathic chronic laryngitis. 


\section{MATERIALS AND METHODS}

Enrollment started in October, 1998, and ended in December, 1999. Patients with chronic idiopathic laryngitis were referred by a single otolaryngologist (P.L.) at the Albuquerque VA Medical Center after a complete examination of the nose, pharynx, and larynx. Idiopathic chronic laryngitis was defined by a combination of the following symptoms and signs: 1) presence of hoarseness, frequent clearing of the throat, dry cough, globus (persistent sensation of a lump in the throat), or persistent sore throat for at least $3 \mathrm{wk} ; 2$ ) grossly visualized laryngeal findings consistent with posterior laryngitis, such as erythema, edema, contact ulcer, or granuloma; and 3) absence of concurrent infectious or allergic causes of laryngitis. Patients with aerodigestive malignancies, radiation therapy, or previous gastroesophageal surgery were excluded. The initial laryngeal symptoms (hoarseness, cough, globus, sore throat), esophageal symptoms (heartburn, acid regurgitation, acid taste in mouth), and the gross laryngeal findings were seen on telescopic video laryngoscopy. The laryngeal lesions were located at the arytenoid and interarytenoid area of the larynx, including the vocal cords. These lesions were recorded as follows: 1) erythema, visual impression of reddening compared to the supraglottic mucosa; 2) edema, swollen contours of the cartilage; 3 ) granuloma, nonmalignant reactive excess tissue on the mucosa; and 4) ulcer, discontinuity of mucosa on the laryngeal surface of the arytenoid where white cartilage was seen on endoscopy. The information was systematically and prospectively recorded in a data acquisition form designed for the purpose of the study.

All patients underwent an upper GI endoscopy performed by the same gastroenterologist who measured and recorded the distance between the proximal end of the lower esophageal sphincter (LES) and the incisor teeth. Immediately after upper endoscopy, a 24-h esophageal $\mathrm{pH}$ catheter with two $\mathrm{pH}$ electrodes separated by $15 \mathrm{~cm}$ was introduced transnasally into the esophagus. Outside the study protocol, eight patients underwent motility studies in which the location of the LES was determined manometrically and recorded. The distal probe was placed $5 \mathrm{~cm}$ above the LES as determined by a combination of $\mathrm{pH}$ step-up technique, endoscopic visualization, motility studies when available, and visualization under fluoroscopy. The proximal probe, $15 \mathrm{~cm}$ higher than the distal one, was located distal to the upper esophageal sphincter (UES). The probe was connected to a dual-channel Digitrapper recording system (Synectics, Irving, TX), which samples $\mathrm{pH}$ at 5-s intervals. The information stored in the Digitrapper was digitally downloaded into a PC with software capabilities to analyze episodes of acid reflux at the level of each probe. Histamine-2 receptor antagonist and proton pump inhibitor drug therapy was discontinued for at least $2 \mathrm{wk}$ before upper endoscopy and 24-h pH-metry. A DeMeester score $>14.7$ was used as the criterion for the presence of pathological acid reflux at the distal probe. Acid reflux at the proximal probe was recorded and reported as the percentage of time with $\mathrm{pH}<4$. Informed written consent was obtained from all patients who participated. The presence of esophagitis was noted and graded according to the Los Angeles classification of esophagitis: 1) one or more mucosal breaks confined to the mucosal folds, each no longer than $5 \mathrm{~mm}$; 2) at least one mucosal break longer than $5 \mathrm{~mm}$ confined to the mucosal folds but not continuous between the tops of two mucosal folds; 3 ) at least one mucosal break continuous between the tops of two mucosal folds but not circumferential; and 4) circumferential mucosal breaks (12). The study protocol was approved by the Human Research Review Committee of the University of New Mexico and the Research Committee of the Albuquerque VA Medical Center.

\section{Intervention}

Patients were randomly assigned to receive either lansoprazole 30-mg oral capsules b.i.d. before meals or matching placebo capsules b.i.d. Both lansoprazole and placebo were given for a total of 3 months. The randomization process was computer generated in blocks of four. Allocation of the treatment was concealed, and patients and investigators were blinded as to the treatment status. Laryngeal as well as esophageal symptom questionnaires were readministered 6 wk and 3 months after the start of treatment. Pill counting was performed at each visit to check for compliance with treatment. Patients were allowed to continue using their previous medications for nongastric acid-related disorders. A repeat laryngeal examination was performed by the same otolaryngologist at the end of treatment (3 months). Upper endoscopy and ambulatory 24-h esophageal $\mathrm{pH}$-metry were repeated at 3 months if there were abnormal findings at the baseline examination. Complete symptomatic response was defined by the total resolution of all presenting symptoms of laryngitis. Nonresponse to therapy was defined by persistence of any of the initial laryngitis symptoms. Complete resolution of laryngeal signs was defined by the absence of all abnormal signs noted on pretreatment evaluation, whereas partial resolution denoted the resolution of some but not all of the abnormal findings.

\section{Statistical Analyses}

Patients treated with lansoprazole were compared with those treated with placebo using several sets of univariate analyses. Similarly, complete symptomatic responders at the end of the study were compared with nonresponders using univariate analyses. Pearson's $\chi^{2}$ tests were used for dichotomous variables, whereas $t$-tests were used for continuous variables. All tests were two-tailed. Differences between complete response rates in the lansoprazole and placebo groups were analyzed using both intention-to-treat (ITT) and per-protocol analyses. A power calculation indicated that 11 patients needed to be enrolled in each group to detect a $70 \%$ difference in efficacy between $10 \%$ assumed response with placebo and $80 \%$ response rate with lansoprazole treatment (5\% $\alpha$-error and $20 \% \beta$-error). 
Table 1. Baseline Characteristics in Patients with Idiopathic Chronic Laryngitis

\begin{tabular}{lcc}
\hline \multicolumn{1}{c}{ Variable } & $\begin{array}{c}\text { Lansoprazole } \\
(\mathrm{n}=12)\end{array}$ & $\begin{array}{c}\text { Placebo } \\
(\mathrm{n}=10)\end{array}$ \\
\hline Mean age in yr (SD) & $59(12)$ & $65(12)$ \\
Men & $12(100 \%)$ & $9(90 \%)$ \\
Tobacco smoking (active) & $1(8 \%)$ & $1(10 \%)$ \\
Alcohol drinking & $2(17 \%)$ & $4(40 \%)$ \\
GERD symptoms & & \\
$\quad$ Heartburn & $6(50 \%)$ & $8(80 \%)$ \\
Regurgitation & $5(42 \%)$ & $6(60 \%)$ \\
Acid taste in mouth & $4(34 \%)$ & $5(50 \%)$ \\
Erosive esophagitis & $3(27 \%)$ & $1(10 \%)$ \\
Laryngeal symptoms & & \\
Sore throat & $7(58 \%)$ & $5(50 \%)$ \\
Globus & $8(67 \%)$ & $4(40 \%)$ \\
Frequent throat clearing & $10(83 \%)$ & $6(60 \%)$ \\
Postnasal drip & $5(42 \%)$ & $5(50 \%)$ \\
Cough & $9(71 \%)$ & $5(50 \%)$ \\
Laryngeal signs & & \\
Erythema & $7(58 \%)$ & $8(80 \%)$ \\
Edema & $8(67 \%)$ & $5(50 \%)$ \\
Granuloma & $1(9 \%)$ & $0(0 \%)$ \\
Contact ulcer & $5(42 \%)$ & $7(70 \%)$ \\
\hline
\end{tabular}

Patients were randomized to receive either lansoprazole $30 \mathrm{mg}$ b.i.d. or placebo. GERD = gastroesophageal reflux disease.

\section{RESULTS}

A total of 27 patients were referred with the diagnosis of chronic idiopathic laryngitis as defined in Materials and Methods. All patients underwent upper GI endoscopy and ambulatory 24-h esophageal pH-metry. Five patients subsequently declined enrollment into the study, whereas the remaining 22 patients were randomly assigned to receive either lansoprazole $30 \mathrm{mg}$ b.i.d. or a matching placebo b.i.d. The baseline characteristics of the study population are shown in Table 1 . There were no statistically significant differences between the two groups with regards to age, gender, ethnicity, smoking, presence of GERD symptoms, laryngeal symptoms, initial presence of esophagitis, or laryngeal signs. In addition, there were no differences in the results of the 24-h esophageal $\mathrm{pH}$-metry values obtained at baseline. Five patients (42\%) in the lansoprazole group and seven $(70 \%)$ in the placebo group had a DeMeester score of $>14.7$ recorded at the distal $\mathrm{pH}$ probe. On the other hand, only two patients (17\%) in the lansoprazole groups and three $(30 \%)$ in the placebo group had a $\mathrm{pH}<4$ for $>1 \%$ of the time recorded at the proximal esophageal probe during the 24-h esophageal $\mathrm{pH}$-metry.

Of the 22 patients randomized, 20 finished the study and had a complete set of information at baseline and at 3 months, 11 of whom received lansoprazole and nine received placebo. Two patients dropped out of the study, one patient in the placebo group did not return for follow-up, and one on lansoprazole discontinued the medicine because of nonspecific abdominal complaints. These two patients were included in the ITT analysis.

At the end of 3 months of treatment, a total of seven patients were complete responders as defined by the resolution of all of presenting laryngeal symptoms. Six of these complete responders were treated with lansoprazole and one with placebo.

We performed both ITT and per-protocol analyses to compare patients in the lansoprazole group with those in the placebo group. In the ITT analysis, the rate of complete symptomatic responders in the lansoprazole group (six of $12,50 \%$ ) was significantly higher than the response rate in the placebo group (one of $10,10 \%$ ) (Pearson's $\chi^{2}$ test 4.023 , $p=0.0449)$. In ITT, the study was $66 \%$ powered to detect a difference between $10 \%$ in the placebo group and $50 \%$ in the lansoprazole group with an alpha error of $5 \%$. In the per-protocol analysis, which was restricted to 20 patients who completed the study, the rate of complete symptomatic responders in the lansoprazole-treated (six of 11,55\%) and placebo-treated (one of 9,11\%) groups increased further (Pearson's $\chi^{2} 4.105, p=0.0428$ ).

By the end of 3 months of treatment, seven patients (58\%) in the lansoprazole group had either complete $(n=2)$ or partial $(n=5)$ resolution of their laryngeal signs. A smaller proportion $(30 \%)$ of placebo-treated patients had partial resolution $(\mathrm{n}=3)$, and none had complete resolution of laryngeal signs. These differences, however, did not reach statistical significance $(p=0.123)$.

Despite complete symptomatic response, laryngeal signs did not fully recover in most patients. Among the seven complete symptomatic responders, only one patient had complete resolution of the abnormal laryngeal signs (erythema and edema), which were noted on baseline examination, three had partial improvement, and three had no change of their laryngeal signs. Among patients who were not complete symptomatic responders $(n=15)$, three had partial resolution and the rest had no resolution of laryngeal signs.

In Table 2, a comparison is made between patients with complete symptomatic response $(\mathrm{n}=7)$ and the rest of the study population $(\mathrm{n}=15)$. Lansoprazole treatment was the only significant predictor of response, $(86 \%$ vs $40 \%, p=$ $0.04)$. None of the baseline demographic features, presence of GERD signs or symptoms, and laryngeal signs or symptoms were significantly different between the two groups. Two patients in each group had erosive esophagitis, two grades $\mathrm{C}$ in the responders groups, one $\mathrm{B}$, and one $\mathrm{C}$ in the nonreponders. In addition, there were no significant differences in the baseline 24-h pH-metry studies. Only one complete responder and four nonresponders had a proximal esophageal $\mathrm{pH}<4$ for $>1 \%$ of the time. Three complete symptomatic responders $(43 \%)$ and seven nonresponders $(47 \%)$ had a DeMeester score $>14.7$ recorded at the distal esophageal $\mathrm{pH}$ probe. There was no significant difference in the mean percent time with $\mathrm{pH}<4$ between responders and nonresponders. Responders had two to 118 daily episodes of distal esophageal acid reflux (mean 51.7, median 39) and none to two reflux episodes $>5$ min (mean 0.7 , median 0 ), which was not statistically different from nonresponders 
Table 2. Comparison Between Patients With Complete Symptomatic Response and the Rest of the Study Population

\begin{tabular}{lcr}
\hline \multicolumn{1}{c}{ Variable } & $\begin{array}{c}\text { Complete } \\
\text { Responders } \\
(\mathrm{n}=7)\end{array}$ & $\begin{array}{c}\text { Noncomplete } \\
\text { Responders } \\
(\mathrm{n}=15)\end{array}$ \\
\hline $\begin{array}{l}\text { Treatment with lansoprazole } 30 \\
\quad \text { mg b.i.d. }{ }^{*}\end{array}$ & $6(86 \%)$ & $6(40 \%)$ \\
GERD symptoms & $4(57 \%)$ & $12(80 \%)$ \\
Erosive esophagitis & $2(29 \%)$ & $2(13 \%)$ \\
Percent time in 24-h distal & $6.6( \pm 5.7)$ & $2.5( \pm 2.5)$ \\
$\quad$ esophageal pH $<4$ & & \\
Distal 24-h esophageal pH $>14.7$ & $3(43 \%)$ & $9(60 \%)$ \\
Proximal 24-h esophageal pH $>0$ & $4(57 \%)$ & $12(80 \%)$ \\
Proximal 24-h esophageal pH $>1$ & $1(14 \%)$ & $4(27 \%)$ \\
Laryngeal edema & $2(29 \%)$ & $10(67 \%)$ \\
Laryngeal erythema & $4(57 \%)$ & $10(67 \%)$ \\
Laryngeal ulcer & $4(57 \%)$ & $8(53 \%)$ \\
\hline Comper
\end{tabular}

Complete response was defined by the resolution of all presenting laryngeal symptoms.

$* p=0.04$. With the exception of $*$, all differences were not statistically significant.

GERD = gastroesophageal reflux disease

who had 21 to 204 reflux episodes (mean 98, median 79) and none to 28 reflux episodes $>5 \min (p=0.8$ and $p=0.05$, respectively). Similarly, no significant differences were seen in proximal $\mathrm{pH}$ reading between the two groups. Among responders, proximal $\mathrm{pH}$-metry revealed none to 37 episodes of acid reflux (mean 13, median 5) and no reflux episodes $>5 \mathrm{~min}$. Nonresponders had none to 40 episodes of acid reflux recorded at the proximal $\mathrm{pH}$ probe, and two patients had one and four episodes of acid reflux $>5$ min. Because of the small number of complete symptomatic responders, multivariate assessment of potential predictors of response was not possible.

\section{DISCUSSION}

In this randomized, placebo-controlled trial, lansoprazole 30 $\mathrm{mg}$ b.i.d. for 3 months resulted in complete resolution of laryngeal symptoms in 50\% of patients with chronic laryngitis. This was significantly higher than the $10 \%$ resolution among patients treated with placebo. Among complete symptomatic responders, none of the initial diagnostic tests employed were significantly different from nonresponders. These tests, including GERD symptoms, ambulatory distal or proximal $24-\mathrm{h}$ esophageal $\mathrm{pH}$, laryngeal examination, or GI endoscopy, were predictive of eventual response. These results suggest that among patients with chronic laryngitis in whom significant infectious and allergic causes are excluded, empirical therapy with acid suppression treatment is the best initial management strategy.

Several previous uncontrolled studies have indicated a potential benefit for treating GERD among patients with laryngitis. The present study, however, provides the only evidence from a placebo-controlled study supporting the efficacy of gastric acid suppression in treating chronic laryngitis. In the only placebo-controlled study previously performed, no benefit could be shown for treating eight patients with posterior laryngopharyngitis with lansoprazole $30 \mathrm{mg}$ b.i.d. for 3 months compared with seven patients who were given placebo (13). In this small sample, improvements in symptoms were recorded as a mean value of a composite laryngeal symptom score for all patients. This did not include examination of responses in individual patients, thus allowing the possibility that a few outlying observations could significantly influence the overall results (13).

Among patients with idiopathic chronic laryngitis, treatment with lansoprazole $30 \mathrm{mg}$ b.i.d. was the only predictor of complete response. Despite the relatively small sample size of the present study, the difference in the rate of complete responders was striking allowing the detection of differences with $99 \%$ power. However, the small sample size did not allow for concomitant adjustment for potential confounders. In addition, although significant differences could not be detected in initial baseline characteristics of patients or predictors of response, a type II $(\beta)$ error resulting from the small sample size could have obscured differences in some of these parameters.

In measuring the 24-h esophageal $\mathrm{pH}$, we followed a previously described method of placing the distal $\mathrm{pH}$ probe $5 \mathrm{~cm}$ above the LES with the proximal probe $15 \mathrm{~cm}$ higher than the distal one and just below the UES (5). A certain degree of variability in placement might have affected the location of the proximal probe because of differences in the lengths of the esophagus in subjects. Such minor differences are unlikely to affect the reported results. Previous studies of normal proximal esophageal 24-h $\mathrm{pH}$ found $\mathrm{pH}<4$ during a narrow range between 0 and $1 \%$ of the time whether the probe was placed below, above, or at the UES (5, 14-16).

In the absence of erosive esophagitis, the diagnosis of GERD can be difficult. Classic GERD symptoms such as heartburn and acid regurgitation have low sensitivity, 36\% and 6\%, respectively, for diagnosing GERD (17). Ambulatory 24-h esophageal pH-metry is considered the gold standard test for classic GERD, with a reported sensitivity between $79 \%$ and $90 \%(18-19)$. However, the diagnosis of GERD-related extra-esophageal manifestations, such as laryngitis, is more challenging, as one has to first diagnose GERD and then prove causality between GERD and its presumed complication. At the present time, apart from a favorable response of laryngitis to acid suppressive therapy, there is no accepted test used to diagnose GERD-related laryngitis. The role of proximal esophageal $\mathrm{pH}$ monitoring in predicting response of laryngitis to acid-suppressive therapy is still poorly defined. Although the presence of acid reflux in the proximal esophagus might be suggestive of the presence of GERD-related laryngitis, its sensitivity and reproducibility are too low (55\%) for it to be used as an initial diagnostic test for GERD-related laryngitis $(14,20)$.

The relative ease of administering a proton pump inhibitor makes it an attractive initial therapy in managing suspected GERD-related laryngitis. Several investigators suggested using the symptomatic response to high-dose proton 
pump inhibitors as a diagnostic tool for $\operatorname{GERD}(21,22)$ and GERD related extra-esophageal complications such as laryngitis (10), noncardiac chest pain (23), and chronic cough (24). For example, Wo et al. (10) reported a favorable response to an empiric therapeutic trial of omeprazole $40 \mathrm{mg}$ for $8 \mathrm{wk}$ in $14(67 \%)$ out of 21 patients with posterior laryngitis. In a placebo-controlled, cross-over study, Fass et al. (22) examined the use of omeprazole $60 \mathrm{mg} / \mathrm{day}$ for 1 wk, in diagnosing GERD-related chest pain. This study reported high sensitivity $(78 \%)$ and specificity $(86 \%)$ of the omeprazole diagnostic trial compared to a combination of 24-h esophageal pH-metry and upper GI endoscopy.

In summary, this study provides the first case-by-case evidence from a randomized, placebo-controlled study to indicate that empirical gastric acid suppression with lansoprazole provides significant benefit to patients with idiopathic chronic laryngitis. These results were obtained from a selected referral population with a high likelihood of GERD and may not be generalized to patients with laryngitis in a primary care setting. Future large multicenter trials are warranted to identify clinically useful diagnostic features, and predictors of response to treatment of patients with laryngitis.

\section{ACKNOWLEDGMENT}

This study was partially supported by a grant from Tap Pharmaceuticals.

Reprint requests and correspondence: Hashem B. El-Serag, M.D., M.P.H., The Houston Veterans Affairs Medical Center (152), 2002 Holcombe Boulevard, Houston, TX 77030.

Received Aug. 10, 2000; accepted Dec. 4, 2000

\section{REFERENCES}

1. El-Serag HB, Sonnenberg A. Comorbid occurrence of laryngeal or pulmonary disease with esophagitis in United States Veterans. Gastroenterology 1997;113:755-60.

2. Koufman JA. The otolaryngologic manifestations of gastroesophageal reflux disease (GERD): A clinical investigation of 225 patients using ambulatory 24-hour $\mathrm{pH}$ monitoring and an experimental investigation of the role of acid and pepsin in the development of laryngeal injury. Laryngoscope 1991;101:165 .

3. Richter JE. Extraesophageal presentations of gastroesophageal reflux disease. Semin Gastrointest Dis 1997;8:75-89.

4. Young JL, Shaw GY, Searl JP, et al. Laryngeal manifestation of gastroesophageal reflux disease: Endoscopic appearance and management. Gastrointest Endosc 1995;43:225-30.

5. Wiener GJ, Koufman JA, Wu WC, et al. Chronic hoarseness secondary to gastroesophageal reflux disease: Documentation with $24-\mathrm{H}$ ambulatory $\mathrm{pH}$ monitoring. Am J Gastroenterol 1989;84:1503-8.
6. Wilson JA, White A, Haacke NP, et al. Gastroesophageal reflux disease and posterior laryngitis. Ann Otol Rhinol Laryngol 1989;98:405-10.

7. Kamel PL, Hanson D, Kharilas PJ. Omeprazole for the treatment of posterior laryngitis. Am J Med 1994;96:321-6.

8. Kamel PL, Hanson DG, Kharilas PJ. Outcomes of antireflux therapy in the treatment of chronic laryngitis. Ann Otol Rhinol Laryngol 1995;104:550-5.

9. Waring JP, Lacayo L, Hunter J, et al. Chronic cough and hoarseness in patients with gastroesophageal reflux disease. Dig Dis Sci 1995;40:1093-7.

10. Wo JM, Grist WJ, Gussack G, et al. Empiric trial of high-dose omeprazole in patients with posterior laryngitis: A prospective study. Am J Gastroenterol 1997;92:2160-5.

11. Japerson D, Weber R, Draf W, et al. Omeprazole for the treatment of reflux associated chronic laryngitis. J Gastroenterol 1996;31:765-7.

12. Armstrong D, Bennet JR, Blum AL, et al. The endoscopic assessment of esophagitis: A progress report on observer agreement. Gastroenterology 1996;111:85-92.

13. Havas T, Huang S, Levy M, et al. Posterior pharyngolaryngitis - double-blind randomized placebo-controlled trial of proton pump inhibitor therapy. Aust J Otolaryng 1999;3:243-6.

14. Vaezi MF, Schroeder PL, Richter JE. Reproducibility of proximal $\mathrm{pH}$ parameters in 24-hour ambulatory esophageal $\mathrm{pH}$ monitoring. Am J Gastroenterol 1997;92:825-9.

15. Smit CF, Tan J, Devriese PP, et al. Ambulatory pH measurements at the upper esophageal sphincter. Laryngoscope 1998; 108:299-302.

16. Dobhan R, Castell DO. Normal, and abnormal proximal esophageal acid exposure: Results of ambulatory dual-probe pH monitoring. Am J Gastroenterol 1993;88:25-9.

17. Klauser AG, Schindlbeck NE, Muller-Lissner SA. Symptoms in gastro-oesophageal reflux disease. Lancet 1990;335(8683): 205-8.

18. Euler AR, Byrne WJ. Twenty four hour esophageal intraluminal $\mathrm{pH}$ probe testing: A comparative analysis. Gastroenterology 1981;80:957-61.

19. Jamieson JR, Sten HJ, DeMeester TR, et al. Ambulatory 24-hour esophageal $\mathrm{pH}$ monitoring: Normal values, optimal thresholds, specificity, sensitivity, and reproducibility. Am J Gastroenterol 1992;87:1102-11.

20. Richter JE, Hicks DM. Unresolved issues in gastroesophageal reflux-related ear, nose, and throat problems. Am J Gastroenterol 1997;92:2143-4.

21. Schenk BE, Kuipers EJ, Klinkenberg-Knol EC, et al. Omeprazole as a diagnostic tool in gastroesophageal reflux disease. Am J Gastroenterol 1997;92:1997-2000.

22. Fass R, Ofman JJ, Gralnek IM, et al. Clinical and economic assessment of the omeprazole test in patients with symptoms suggestive of gastroesophageal reflux disease. Arch Intern Med 1999;159:2161-8.

23. Fass R, Fennerty MB, Ofman JJ, et al. The clinical and economic value of a short course of omeprazole in patients with noncardiac chest pain. Gastroenterology 1998;115: 42-9.

24. Ours TM, Kavuru MS, Schilz RJ, et al. A prospective evaluation of esophageal testing and a double-blind, randomized study of omeprazole in a diagnostic and therapeutic algorithm for chronic cough. Am J Gastroenterol 1999;94:3131-8. 